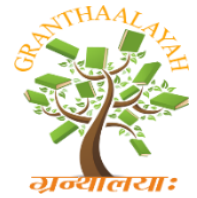

\author{
INTERNATIONAL JOURNAL OF RE
GRANTHAALAYAH \\ A knowledge Repository
}

Science

\title{
ELECTRONIC, STRUCTURAL AND PHARMACOCINETIC CHARACTERIZATION OF TRICYCLIC ALKALOID ALTERNAMIDE A: A SEMI-EMPIRICAL QUANTUM STUDY AND ADMET
}

\author{
Sandy Pereira Estácio ${ }^{1}$, Francisco Rogênio Da Silva Mendes ${ }^{1}$, Emanuelle Machado \\ Marinho $^{2}$, Othon Souto Campos ${ }^{3}$, Márcia Machado Marinho², Emmanuel Silva Marinho*1 \\ ${ }^{1}$ Chemistry Department, State University of Ceará, Brazil \\ ${ }^{2}$ Chemistry Department, Federal University of Ceará, Brazil \\ ${ }^{3}$ Chemistry Department, Federal University of Espirito Santo, Brazil
}

\begin{abstract}
Chagas disease is one of the biggest socioeconomic problems in Latin America. Caused by the protozoan parasite Trypanosoma cruzi, affecting 7 million people, causing approximately 14,000 deaths per year. Alternamide, a tricyclic alkaloid present in Alternanthera littoralis, an herbaceous plant found on beaches of the Brazilian its extracts are used in traditional medicine for treatment of infectious and inflammatory diseases, which showed anti Trypanocida activity. In this context, in the present work we present the results of the electronic, structural and pharmacokinetic characterization study of the promising phytopharmaceutical Alternamide A. Using the semiempirical quantum formalism it was possible to identify the most stable conformation, boundary orbitals, calculate to identify nucleophilic sites and reactivity descriptors. Through in silico absorption, distribution, metabolism, excretion and toxicity (ADMET) simulations, including solubility, blood-brain barrier (BHE), plasma protein binding, CYP2D6 binding, gastrointestinal absorption and hepatotoxicity, it was observed that good oral bioavailability and high-water solubility high gastrointestinal absorption. The synthetic accessibility score was 2.75 , which means that it would be easy to synthesize the molecule under study. Highlighting what this study represents is a key step for future molecular docking and drug design studies for the development of inhibitors of the evolutionary forms of the molecule T-crossed.
\end{abstract}

Keywords: Alkaloid; Chagas disease; Neglected disease; Quantum study; Semi-empirical; Trypanosoma cruzi.

Cite This Article: Sandy Pereira Estácio, Francisco Rogênio Da Silva Mendes, Emanuelle Machado Marinho, Othon Souto Campos, Márcia Machado Marinho, and Emmanuel Silva Marinho. (2019). "ELECTRONIC, STRUCTURAL AND PHARMACOCINETIC CHARACTERIZATION OF TRICYCLIC ALKALOID ALTERNAMIDE A: A SEMIEMPIRICAL QUANTUM STUDY AND ADMET." International Journal of Research Granthaalayah, 7(10), 429-447. https://doi.org/10.29121/granthaalayah.v7.i10.2019.417. 


\section{Introduction}

Discovered in 1909 by Brazilian doctor Carlos Chagas, Chagas disease is one of the biggest socioeconomic problems in Latin America. It is a zoonosis that affects about 7 million people, which causes approximately 14,000 deaths per year [1]. The causative agent of Chagas disease is Trypanosoma cruzi, a flagellated protozoan that in its evolutionary cycle must pass through it hosts of various classes of mammals, including man or even some insects that are known as barbeiro and so the large numbers of infections occurrence [2][3].It is also one of the neglected diseases, so this pathology does not arouse much interest in the pharmaceutical industries due to the low financial return. Chagas disease chemotherapy is restricted to only two nitroheterocycle drugs, nifurtimox and benznidazole. Therefore, it is of great importance, given the severity and scope of the pathology, to strengthen the research for new trypanomycid agent candidates in their treatment.[4] [5][6][7].

Alternamide A (7,8-dihydroxy-1,2,4,5-tetrahydro-3H-1,5-ethano [c] azepin-3-one), a tricyclic alkaloid isolated from the Alternanthera littoralis P. Beauvis, a herbaceous plant commonly found on beaches of the Brazilian eastern coast and its extracts are used in traditional medicine for treatment of infectious and inflammatory diseases, which presented antiprotozoal activity was assayed against trypomastigote forms of Trypanosoma cruzi and amastigotes of Leishmania amazonenses[8].

Through molecular modeling, we can determine parameters that relate structure and activity [9]. For this, molecular modeling is used, which can be defined as a software system and tools that allow the construction, editing, visualization and analysis of molecular structures. Thus, the computational chemistry allows the complete characterization of the compounds, generating structures with high fidelity rate to native structures and stable conformational geometry, as well as some relevant indices for drug planning. Such as: formation heat, minimum potential energy, homo and lumo energies, dipole moment and the specific arrangement of each atom in the molecule [10] [11] [12]. In addition to electronic and structural characterization, computer modeling allows through models to simulate the behavior of substances in various media, especially with respect to absorption, distribution, metabolism, excretion and toxicity (ADMET) in the human body. The use of algorithms for ADMET simulation are highlighting the discovery and design of new drugs, being applied at an early stage in the research process, promoting both financial and ethical aspects, as well as reducing the initial laboratory cost and use of animals in the development of new medicines [13] [14] [15].

In this context, in the present work, we present the results of the electronic, structural and pharmacokinetic characterization study of the promising phytopharmaco Alternamide A, with initial support for future molecular docking and drug design studies for the development of inhibitors of $T$-cruzi evolutionary forms. 


\section{Materials and Methods}

\section{Computational Details}

All the computations were performed on Dell Inspiron personal computer with intel® Core TM i7-4510U processor, 16 GB RAM, 2GB AMD Radeon ${ }^{\circledR}$ video card and Microsoft Windows $8.1{ }^{\circledR}$ as operating system. All codes used are free license for academic use.

\section{Structural Optimization}

Geometry optimization is a technique that aims to find a set of coordinates that minimize the potential energy of the system under study [16]. The basic procedure is to move over the potential surface in the direction in which the energy decreases so that the system is brought to a near local minimum energy. Power minimization makes use of only a small portion of the configuration space. But by adjusting atomic positions, it relaxes distortions in chemical bonds, bond angles, bond lengths, and torsion angles [16][17].

The study of the Alternamide A molecule was carried out using MarvinSket $@$ [18] and ArgusLab® Version 4.0[19] configured to perform semi-empirical calculations (based on the theory of quantum mechanics with Hamiltonian PM3 (Parametric Method 3)[20], removing some of its molecular characteristics, the Avogadro [21] program was also used to determine the properties of atoms, properties of bonds, angle properties and torsional properties of atoms[22][23][24].

\section{Fronteir Orbitals, MESP And Descriptores Reactivity}

Electrostatic potential surface maps (MESP) are generated after the overlap in the molecule of a positively charged particle that travels over the van der Waals contact surface and by revealing a repulsion region, represents the positive, white-colored potential, and a region of attraction represents the negative potential of red color, based on equation 1[25][26].

$$
V(r)=\sum_{A} \frac{Z_{\mathrm{A}}}{\left|\overrightarrow{R_{\mathrm{A}}}-\overrightarrow{\vec{r}}\right|}-\int \frac{\rho(\vec{r})}{\left|\overrightarrow{r^{\prime}}-\vec{r}\right|} d r^{\prime}
$$

Since $\mathrm{Z}$ is the charge of nucleus $\mathrm{A}$, located at $\mathrm{R}, \mathrm{A} \rho \mathrm{A}(\mathrm{R})$ is the electron density function for the molecule. $\mathrm{V}(\mathrm{r})$ is the resulting electrostatic liquid effect produced at the $\mathrm{r}$ point by both electrons and nuclei of the molecule. Since the first term represents the contributions as a function of electron potential and the second term as a function of the nuclei[25].

Using the formalism of molecular orbital theory, we can learn from the LCAO-MO model [9][26], where the orbitals of each molecule are described as linear combinations of the atomic orbitals of the molecule, we can generate the HOMO and LUMO boundary orbitals (respectively, highest occupied orbital molecular and lowest unoccupied molecular orbital), which may indicate the main electron donation and reception sites, respectively. That is, the number of molecular orbitals formed will always be equal to the number of atomic orbitals involved in their bonding.

A reactivity factor to be analyzed and energy that is influenced influences the orbitals of a molecule on its own orbitals on nearby molecules, this energy is evaluated in the form of energy bands, which have direct dependence on chemical composition and molecular structure [26]. 
The band that comprises the highest and least busy levels of energy is called the conduction band, while the band consisting of the lowest energy occupied levels is called the valence band [27] [26] [28]. The region that exists between the valence band (orbital HOMO) and the conduction band (orbital LUMO) is called the gap, which corresponds to an energetically forbidden region to electrons. These energies can describe the electronic properties of a given molecule, such as its reactivity and the formation of charge transfer complexes. The formation of transition states is due to the interaction between the boundary orbitals of the reactive species [29].

Using the .out file generated by the structure optimization, the boundary orbitals, HOMO and LUMO were plotted, and the MESP was rendered.

To correlate structure and reactivity of molecules the values of the orbitals were used, the values of the orbital frontier were used to calculate the descriptors based on according to Koopman's Theory [30]: Gap, electron affinity (A), electronegativity $(\chi)$, vertical ionization potential (i), chemical hardness $(\eta)$, chemical softness $(s)$, electronic chemical potential $(\mu)$, electrophilicity index $(\omega)$ [31]. To obtain the values, the following formulas were used[32][33] [36][37][32]:

$\begin{array}{ll}\text { GAP } & \Delta \varepsilon=\left|\varepsilon_{\text {HOMO }}\right|-\left|\varepsilon_{\text {LUMO }}\right| \\ \text { eléctron affinity (A) } & \mathrm{A}=-\varepsilon_{\text {LUMO }} \\ \text { Vertical Ionization Potential }(\mathrm{I}) & \mathrm{I}=-\varepsilon_{\mathrm{HOMO}} \\ \text { Eletronegativity }(\chi) & \chi=(\mathrm{I}+\mathrm{A}) / 2 \\ \text { Chemical Hardness }(\eta) & \eta=(\mathrm{I}-\mathrm{A}) / 2 \\ \text { Chemical Softness }(\mathrm{S}) & \mathrm{S}=1 / 2 \eta \\ \text { Eletronic Chemical Potential }(\mu) & \mu=-(\mathrm{I}+\mathrm{A}) / 2 \\ \text { Electrophilicity Index }(\omega) & \omega=\mu^{2} / 2 \eta\end{array}$

\section{In silico ADMET}

The properties of Absorption, Distribution, Metabolization, Excretion and Toxicity (ADMET) including solubility, blood brain barrier (BHE), plasma protein binding, CYP2D6 binding, gastrointestinal absorption and hepatotoxicity were evaluated for in silico action of the Alternamide A compound by homology to other molecules that have already been studied in the human body. The models used to predict ADMET properties in this protocol are derived from various experimental data obtained for other drugs with similar chemical structure [38][39].

Prediction of drug similarity for Alternamide A for pharmacokinetics was performed on the Swiss ADME online platform of the Swiss Institute of Bioinformatics (http://www.sib.swiss). In the Swiss ADMET program package, the polar topological surface (TPSA) was calculated [40]. In addition, lipophilicity was predicted according to Wildman et al. [41], solubility and oral 
bioavailability were assessed according to Lipinski and collaborattes. [42] Analysis was performed to determine whether alternamide A was a cytochrome family isoform inhibitor (P450 CYP) such as CYP1A2 and CYP2D6. In addition to other pharmacokinetic prediction (gastrointestinal absorption, glycoprotein $\mathrm{P}$ and blood brain barrier) and prediction of similarity to drugs such as Ghose and Veber Rules and bioavailability [43]

\section{Results and Discussions}

As for the structure in question, Alternamide A, the MarvinSketch program was used to obtain the initial structure (figure 1) with an easily visible initial conformation, but with potential energy different from its native form and some other physical chemical properties (Table 1) necessary to study the structure in molecular modeling, highlighting the LogP partition coefficient (0.93), its density $(1.371 \pm 0.06 \mathrm{~g} / \mathrm{cm} 3)$, surface tension $(58.9 \pm 3.0$ dyne / cm-1). $)$, the solubility of the structure in water $(0.06 \mathrm{mg} / \mathrm{mL})$ that allowed defining the solvent (polar or non-polar) used in docking or molecular dynamics tests.

Table 1: Physicochemical Properties of Alkaloid Alternamide A

\begin{tabular}{|l|l|l|l|}
\hline Property & Value & Property & Value \\
\hline Solubility in water & $0.06 \mathrm{mg} / \mathrm{mL}$ & Polar Surface Area & $69.56 \AA^{2}$ \\
\hline LogP & 0.93 & Polarizability & $22.36 \AA^{3}$ \\
\hline LogS & -1.4 & Refractivity & $58.29 \mathrm{~m}^{3} \cdot \mathrm{mol}^{-1}$ \\
\hline Molecular Formula & $\mathrm{C}_{12} \mathrm{H}_{13} \mathrm{NO}_{3}$ & Density & $1.371 \pm 0,06 \mathrm{~g} / \mathrm{cm}^{3}$ \\
\hline Superficial tension & $58.9 \pm 3.0 \mathrm{dyne} / \mathrm{cm}$ & Molar Volume & $159.8 \pm 3.0 \mathrm{~cm}^{3}$ \\
\hline Monoisotopic Mass & $219.089543 \mathrm{Da}$ & Refractive index & $1.639 \pm 0.02$ \\
\hline Donors \#H & 3 & \#H Receivers & 3 \\
\hline
\end{tabular}

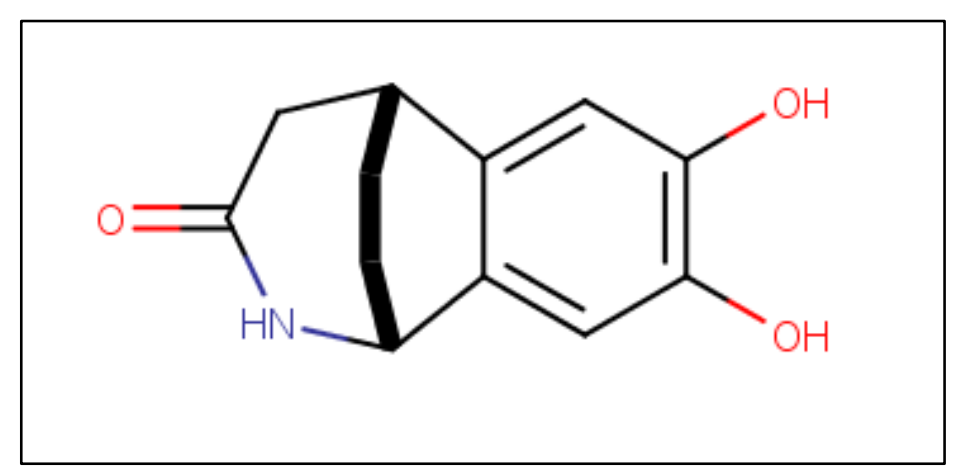

Figure 1: Alternamide A initial structure

Semiempirical methods have become a solution for modeling medium-sized molecules, as they use experimentally pre-established parameters, reducing the number of integrals to be solved for the Schrödinger equation solution (Equation 10)[44][45].

$-\frac{\hbar^{2}}{2 m} \nabla^{2} \Psi+E_{p} \Psi=i \hbar \frac{\partial \Psi}{\partial t}$ 
Since Ep is the potential energy in the region considered, $\mathrm{m}$ is the particle mass associated with this wave function and $\partial \psi / \partial \mathrm{t}$ represents the partial derivative of the wave function in time order; $2 \Psi$ is the Laplacian mathematical operator of $\Psi$. Using the Born-Oppenheimer approximation, which decouples the electronic and nuclear movements, assuming a fixed position of the nuclei, the Schrödinger equation for the Alternamide A molecule was solved, having as a parameter the global energy state, where after a conformational analysis screnning was obtained the configuration that can be considered more thermonidically stable, which presented a selfconsistent field energy (SCF) in the order of 3544,78775 eV (Figure 2), emphasizing that the selfconsistent field theory, based on the assumption that the potential acting on each electron and due to the nuclei and the average charge distribution of the other electrons [46]. In order to characterize the behavior of the molecule against several solvents, the dipolar moment vector was calculated, using electronegativity values relative to each atom, calculates through the resulting vector, which globally calculates the magnitu of the charge displacement of each atom involved in the bond [47]. The alternamide molecule presented a dipole moment vector that showed values in the Cartesians of 0.84969627 ( $\mathrm{X}$ axis), 2.31794357 ( $\mathrm{Y}$ axis) and $\mathrm{Z}$ the value of -2.79671270 ( $\mathrm{Z}$ axis), thus indicating a high polarity in the molecule, it is preferably soluble in polar solvents. With geometric optimization, the theoretically most stable molecule, it is also possible to calculate the formal and partial charges of each atom as well as its valence, despite the neutrality through optimization, it is possible to observe in the results obtained (table 2) the existence of charges partial (residual) charges that come from the electrons are closer or farther from one of the atoms of the bond, carrying charge. Note that carbon has a valence variation of 3 and 4, oxygen 1 and 2 and hydrogen has a valence of 1 .

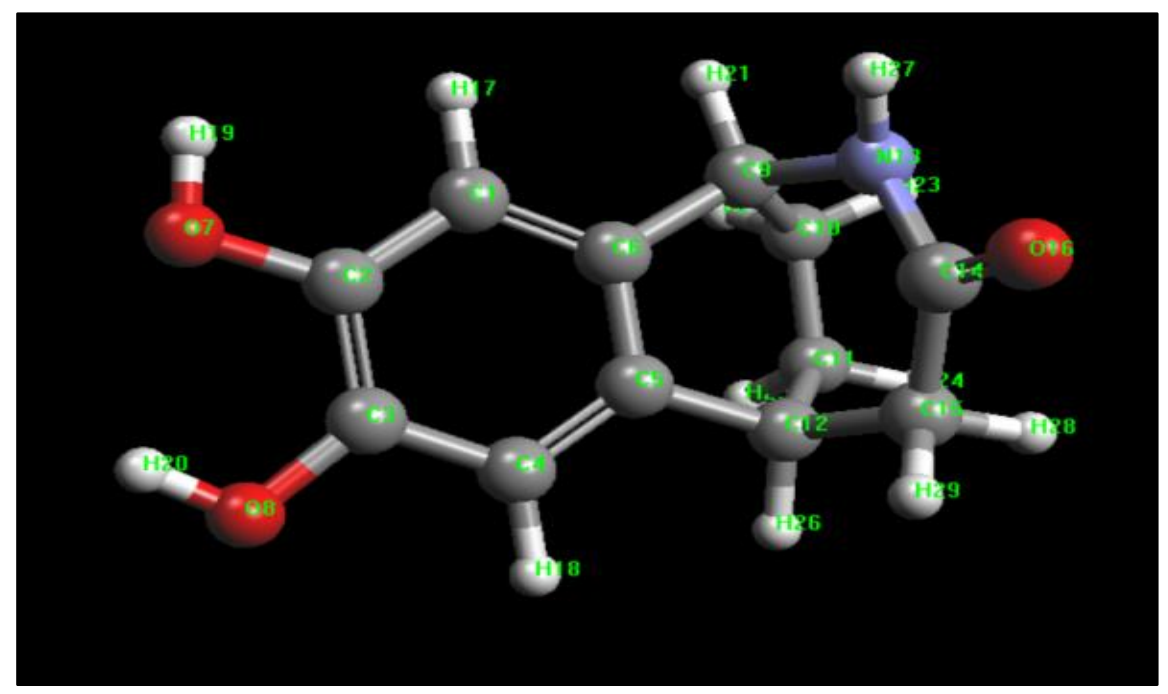

Figure 2: Optimized structure of Alternamide A tricyclic alkaloid

Table 2: Atomic properties of alternamide A

\begin{tabular}{|l|l|l|l|l|}
\hline Atom & \multicolumn{2}{|l|}{ Elemento/type } & Valence & Partial charge \\
\hline 1 & $\mathrm{C}$ & $\mathrm{Car}$ & 3 & -0.011 \\
\hline 2 & $\mathrm{C}$ & $\mathrm{Car}$ & 3 & 0.159 \\
\hline 3 & $\mathrm{C}$ & $\mathrm{Car}$ & 3 & 0.159 \\
\hline 4 & $\mathrm{C}$ & $\mathrm{Car}$ & 3 & -0.013 \\
\hline 5 & $\mathrm{C}$ & $\mathrm{Car}$ & 3 & -0.035 \\
\hline
\end{tabular}




\begin{tabular}{|l|l|l|l|l|}
\hline 6 & C & Car & 3 & -0.020 \\
\hline 7 & O & O3 & 2 & -0.503 \\
\hline 8 & O & O3 & 2 & -0.503 \\
\hline 9 & C & C3 & 4 & 0.049 \\
\hline 10 & C & C3 & 4 & -0.030 \\
\hline 11 & C & C3 & 4 & -0.044 \\
\hline 12 & C & C3 & 4 & -0.007 \\
\hline 13 & N & Nam & 3 & -0.308 \\
\hline 14 & C & C2 & 3 & 0.214 \\
\hline 15 & C & C3 & 4 & 0.031 \\
\hline 16 & O & O2 & 1 & -0.276 \\
\hline 17 & H & H & 1 & 0.066 \\
\hline 18 & H & H & 1 & 0.066 \\
\hline 19 & H & Ho & 1 & 0.292 \\
\hline 20 & H & Ho & 1 & 0.292 \\
\hline 21 & H & H & 1 & 0.055 \\
\hline 22 & H & H & 1 & 0.029 \\
\hline 23 & H & H & 1 & 0.029 \\
\hline 24 & H & H & 1 & 0.027 \\
\hline 25 & H & H & 1 & 0.027 \\
\hline 26 & H & H & 1 & 0.035 \\
\hline 27 & H & H & 1 & 0.149 \\
\hline 28 & H & H & 1 & 0.036 \\
\hline 29 & H & H & 1 & 0.036 \\
\hline & & & & \\
\hline
\end{tabular}

Analyzing the bonds, it was possible to observe a predominance of the predominantly covalent bonds, where we can highlight the bonds between carbon (C4 - C3), (C5 - C6), (C2 - C1) and oxygen-carbon (C11 - O3) as second order bonds, and the bonds between carbon (C8 - C9), (C9 $\mathrm{C} 10),(\mathrm{C} 8-\mathrm{C} 7),(\mathrm{C} 10-\mathrm{C} 12)$ because they are rotatable, as we can see in table 4.

Table 4: Alternamide A binding properties

\begin{tabular}{|l|l|l|l|l|l|l|}
\hline Bond & Type & Start storm & End storm & Bond order & Rotable & Length( $(\mathbf{)}$ \\
\hline 1 & H-C & H9 & C9 & 1 & No & 1.10751 \\
\hline 2 & H-C & H6 & C8 & 1 & No & 1.10738 \\
\hline 3 & C-H & C9 & H8 & 1 & No & 1.10772 \\
\hline 4 & C-C & C9 & C8 & 1 & Yes & 1.52898 \\
\hline 5 & C-C & C9 & C10 & 1 & Yes & 1.5348 \\
\hline 6 & H-C & H10 & C10 & 1 & No & 1.11838 \\
\hline 7 & C-H & C8 & H7 & 1 & No & 1.10775 \\
\hline 8 & C-C & C8 & C7 & 1 & Yes & 1.53845 \\
\hline 9 & H-C & H2 & C4 & 1 & No & 1.09719 \\
\hline 10 & O-H & O2 & H4 & 1 & No & 0.95015 \\
\hline 11 & O-C & O2 & C3 & 1 & No & 1.36922 \\
\hline 12 & C-C & C4 & C3 & 2 & No & 1.40217 \\
\hline 13 & C-C & C4 & C5 & 1 & No & 1.39074 \\
\hline
\end{tabular}




\begin{tabular}{|l|l|l|l|l|l|l|}
\hline 14 & C-C & C3 & C2 & 1 & No & 1.41347 \\
\hline 15 & C-C & C10 & C5 & 1 & No & 1.50161 \\
\hline 16 & C-C & C10 & C12 & 1 & Yes & 1.53300 \\
\hline 17 & C-C & C5 & C6 & 2 & No & 1.39989 \\
\hline 18 & C-O & C2 & O1 & 1 & No & 1.37677 \\
\hline 19 & C-C & C2 & C1 & 2 & No & 1.93354 \\
\hline 20 & O-H & O1 & H3 & 1 & No & 0.95045 \\
\hline 21 & C-C & C6 & C1 & 1 & No & 1.39035 \\
\hline 22 & C-C & C6 & C7 & 1 & No & 1.5052 \\
\hline 23 & C-H & C1 & H1 & 1 & No & 1.09738 \\
\hline 24 & C-H & C7 & H5 & 1 & No & 1.11803 \\
\hline 25 & C-N & C7 & N & 1 & No & 1.49525 \\
\hline 26 & H-C & H12 & C12 & 1 & No & 1.10973 \\
\hline 27 & C-H & C12 & H13 & 1 & No & 1.10936 \\
\hline 28 & C-C & C12 & C11 & 1 & No & 1.51678 \\
\hline 29 & N-C & N & C11 & 1 & No & 1.42795 \\
\hline 30 & N-H & N & H11 & 1 & No & 1.00008 \\
\hline 31 & C-O & C11 & O3 & 2 & No & 1.22503 \\
\hline
\end{tabular}

Regarding the angles, we can highlight the connection angles that were in the range of $105.4833^{\circ}$ $(\mathrm{H}-\mathrm{C} 8-\mathrm{H} 7)$ and $124.5218^{\circ}(\mathrm{C} 2-\mathrm{C} 3-\mathrm{O} 2)\left(\right.$ table 5). The torsion angles were between $-0.0181^{\circ}$ (H2 - C4 - C5 - C6) and -179.9801 ${ }^{\circ}(\mathrm{C} 3$ - C2 - C1 - H1) (Table 6).

Table 5: Alternamide A lead angles

\begin{tabular}{|l|l|l|l|l|l|}
\hline Angle & Type & Start Atom & Vertex & End Atom & Angle $\left(^{\circ}\right)$ \\
\hline 1 & CCC & C2 & C1 & C6 & 119.1211 \\
\hline 2 & CCH & C2 & C1 & H1 & 120.7349 \\
\hline 3 & CCH & C6 & C1 & H1 & 120.1439 \\
\hline 4 & CCO & C3 & C2 & O1 & 117.0555 \\
\hline 5 & CCC & C1 & C2 & C3 & 120.3501 \\
\hline 6 & CCO & C1 & C2 & O1 & 122.5941 \\
\hline 7 & CCO & C4 & C3 & O2 & 115.5915 \\
\hline 8 & CCO & C2 & C3 & O2 & 124.5218 \\
\hline 9 & CCC & C2 & C3 & C4 & 119.8851 \\
\hline 10 & CCH & C3 & C4 & H2 & 120.1964 \\
\hline 11 & CCH & C5 & C4 & H2 & 120.5386 \\
\hline 12 & CCC & C3 & C4 & C5 & 119.2650 \\
\hline 13 & CCC & C4 & C5 & C10 & 122.3942 \\
\hline 14 & CCC & C4 & C5 & C6 & 120.6718 \\
\hline 15 & CCC & C6 & C5 & C10 & 116.9283 \\
\hline 16 & CCC & C1 & C6 & C5 & 120.7060 \\
\hline 17 & CCC & C5 & C6 & C7 & 116.7266 \\
\hline 18 & CCC & C1 & C6 & C7 & 122.5674 \\
\hline 19 & COH & C2 & O1 & H3 & 107.3513 \\
\hline 20 & COH & C3 & O2 & H4 & 108.7348 \\
\hline
\end{tabular}




\begin{tabular}{|l|l|l|l|l|l|}
\hline 21 & $\mathrm{CCC}$ & $\mathrm{C} 6$ & $\mathrm{C} 7$ & $\mathrm{C} 8$ & 110.0866 \\
\hline 22 & $\mathrm{CCH}$ & $\mathrm{C} 8$ & $\mathrm{C} 7$ & $\mathrm{H} 5$ & 108.6699 \\
\hline 23 & $\mathrm{CCN}$ & $\mathrm{C} 8$ & $\mathrm{C} 7$ & $\mathrm{~N}$ & 110.3696 \\
\hline 24 & $\mathrm{CCH}$ & $\mathrm{C} 6$ & $\mathrm{C} 7$ & $\mathrm{H} 5$ & 110.2542 \\
\hline 25 & $\mathrm{CCN}$ & $\mathrm{C} 6$ & $\mathrm{C} 7$ & $\mathrm{~N}$ & 111.3233 \\
\hline 26 & $\mathrm{NCH}$ & $\mathrm{N}$ & $\mathrm{C} 7$ & $\mathrm{H} 5$ & 106.0327 \\
\hline 27 & $\mathrm{CCH}$ & $\mathrm{C} 9$ & $\mathrm{C} 8$ & $\mathrm{H} 6$ & 109.4691 \\
\hline 28 & $\mathrm{HCH}$ & $\mathrm{H} 6$ & $\mathrm{C} 8$ & $\mathrm{H} 7$ & 105.4833 \\
\hline 29 & $\mathrm{CCH}$ & $\mathrm{C} 7$ & $\mathrm{C} 8$ & $\mathrm{H} 6$ & 108.1887 \\
\hline 30 & $\mathrm{CCH}$ & $\mathrm{C} 9$ & $\mathrm{C} 8$ & $\mathrm{H} 7$ & 110.2405 \\
\hline 31 & $\mathrm{CCC}$ & $\mathrm{C} 7$ & $\mathrm{C} 8$ & $\mathrm{C} 9$ & 113.4651 \\
\hline 32 & $\mathrm{CCH}$ & $\mathrm{C} 7$ & $\mathrm{C} 8$ & $\mathrm{H} 7$ & 109.6657 \\
\hline 33 & $\mathrm{HCH}$ & $\mathrm{H} 8$ & $\mathrm{C} 9$ & $\mathrm{H} 9$ & 105.4865 \\
\hline 34 & $\mathrm{CCH}$ & $\mathrm{C} 8$ & $\mathrm{C} 9$ & $\mathrm{H} 9$ & 109.3703 \\
\hline 35 & $\mathrm{CCH}$ & $\mathrm{C} 10$ & $\mathrm{C} 9$ & $\mathrm{H} 9$ & 108.5279 \\
\hline 36 & $\mathrm{CCH}$ & $\mathrm{C} 8$ & $\mathrm{C} 9$ & $\mathrm{H} 8$ & 110.1961 \\
\hline 37 & $\mathrm{CCH}$ & $\mathrm{C} 10$ & $\mathrm{C} 9$ & $\mathrm{H} 8$ & 109.3253 \\
\hline 38 & $\mathrm{CCC}$ & $\mathrm{C} 8$ & $\mathrm{C} 9$ & $\mathrm{C} 10$ & 113.6036 \\
\hline 39 & $\mathrm{CCH}$ & $\mathrm{C} 9$ & $\mathrm{C} 10$ & $\mathrm{H} 10$ & 107.8414 \\
\hline 40 & $\mathrm{CCC}$ & $\mathrm{C} 5$ & $\mathrm{C} 10$ & $\mathrm{C} 9$ & 109.4995 \\
\hline 41 & $\mathrm{CCC}$ & $\mathrm{C} 9$ & $\mathrm{C} 10$ & $\mathrm{C} 12$ & 111.9786 \\
\hline 42 & $\mathrm{CCH}$ & $\mathrm{C} 5$ & $\mathrm{C} 10$ & $\mathrm{H} 10$ & 109.2003 \\
\hline 43 & $\mathrm{CCH}$ & $\mathrm{C} 12$ & $\mathrm{C} 10$ & $\mathrm{H} 10$ & 106.9915 \\
\hline 44 & $\mathrm{CCC}$ & $\mathrm{C} 5$ & $\mathrm{C} 10$ & $\mathrm{C} 12$ & 111.2100 \\
\hline 45 & $\mathrm{CNC}$ & $\mathrm{C} 7$ & $\mathrm{~N}$ & $\mathrm{C} 11$ & 121.1514 \\
\hline 46 & $\mathrm{CNH}$ & $\mathrm{C} 7$ & $\mathrm{~N}$ & $\mathrm{H} 11$ & 111.9097 \\
\hline 47 & $\mathrm{CNH}$ & $\mathrm{C} 11$ & $\mathrm{~N}$ & $\mathrm{H} 11$ & 113.9215 \\
\hline 48 & $\mathrm{NCC}$ & $\mathrm{N}$ & $\mathrm{C} 11$ & $\mathrm{C} 12$ & 122.9328 \\
\hline 49 & $\mathrm{CCO}$ & $\mathrm{C} 12$ & $\mathrm{C} 11$ & $\mathrm{O} 3$ & 121.9509 \\
\hline 50 & $\mathrm{NCO}$ & $\mathrm{N}$ & $\mathrm{C} 11$ & $\mathrm{O} 3$ & 114.9630 \\
\hline 51 & $\mathrm{CCH}$ & $\mathrm{C} 10$ & $\mathrm{C} 12$ & $\mathrm{H} 12$ & 109.3249 \\
\hline 52 & $\mathrm{CCH}$ & $\mathrm{C} 10$ & $\mathrm{C} 12$ & $\mathrm{H} 13$ & 108.7719 \\
\hline 53 & $\mathrm{CCC}$ & $\mathrm{C} 10$ & $\mathrm{C} 12$ & $\mathrm{C} 11$ & 117.1743 \\
\hline 54 & $\mathrm{HCH}$ & $\mathrm{H} 12$ & $\mathrm{C} 12$ & $\mathrm{H} 13$ & 105.7666 \\
\hline 55 & $\mathrm{CCH}$ & $\mathrm{C} 11$ & $\mathrm{C} 12$ & $\mathrm{H} 12$ & 107.5057 \\
\hline 56 & $\mathrm{CCH}$ & $\mathrm{C} 11$ & $\mathrm{C} 12$ & $\mathrm{H} 13$ & 107.7090 \\
\hline & & & & & \\
\hline
\end{tabular}

Table 6: Torsion angles of alternamide A

\begin{tabular}{|l|l|l|l|l|l|l|}
\hline Torsion & Type & Atom 1 & Atom 2 & Atom 3 & Atom 4 & Torsion $\left(^{\circ}\right)$ \\
\hline 1 & HCCH & H9 & C9 & C8 & H6 & -2.3992 \\
\hline 2 & HCCH & H9 & C9 & C8 & H7 & -118.0022 \\
\hline 3 & HCCC & H9 & C9 & C8 & C7 & 118.5569 \\
\hline 4 & HCCH & H8 & C9 & C8 & H6 & 113.1256 \\
\hline 5 & HCCH & H8 & C9 & C8 & H7 & -2.4774 \\
\hline
\end{tabular}


DOI: 10.5281/zenodo.3542089

\begin{tabular}{|c|c|c|c|c|c|c|}
\hline 6 & $\mathrm{HCCC}$ & H8 & C9 & $\mathrm{C} 8$ & $\mathrm{C} 7$ & -125.9183 \\
\hline 7 & $\mathrm{CCCH}$ & $\mathrm{C} 10$ & C9 & $\mathrm{C} 8$ & H6 & -123.8126 \\
\hline 8 & $\mathrm{CCCH}$ & $\mathrm{C} 10$ & C9 & $\mathrm{C} 8$ & $\mathrm{H} 7$ & 120.5843 \\
\hline 9 & $\mathrm{CCCC}$ & $\mathrm{C} 10$ & $\mathrm{C} 9$ & $\mathrm{C} 8$ & $\mathrm{C} 7$ & -2.8566 \\
\hline 10 & $\mathrm{HCCH}$ & H9 & C9 & $\mathrm{C} 10$ & H10 & 45.8811 \\
\hline 11 & $\mathrm{HCCC}$ & $\mathrm{H} 9$ & C9 & $\mathrm{C} 10$ & $\mathrm{C5}$ & -72.8358 \\
\hline 12 & $\mathrm{HCCC}$ & H9 & C9 & $\mathrm{C} 10$ & $\mathrm{C} 12$ & 163.3298 \\
\hline 13 & $\mathrm{HCCH}$ & $\mathrm{H} 8$ & C9 & $\mathrm{C} 10$ & $\mathrm{H} 10$ & -68.6982 \\
\hline 14 & $\mathrm{HCCC}$ & $\mathrm{H} 8$ & C9 & $\mathrm{C} 10$ & $\mathrm{C} 5$ & 172.5849 \\
\hline 15 & $\mathrm{HCCC}$ & $\mathrm{H} 8$ & C9 & $\mathrm{C} 10$ & C12 & 48.7505 \\
\hline 16 & $\mathrm{CCCH}$ & $\mathrm{C} 8$ & C9 & $\mathrm{C} 10$ & $\mathrm{H} 10$ & 167.7639 \\
\hline 17 & $\mathrm{CCCC}$ & $\mathrm{C} 8$ & C9 & $\mathrm{C} 10$ & $\mathrm{C5}$ & 49.0470 \\
\hline 18 & $\mathrm{CCCC}$ & $\mathrm{C} 8$ & C9 & $\mathrm{C} 10$ & $\mathrm{C} 12$ & -74.7875 \\
\hline 19 & $\mathrm{HCCC}$ & H6 & $\mathrm{C} 8$ & $\mathrm{C} 7$ & C6 & 76.4556 \\
\hline 20 & $\mathrm{HCCH}$ & H6 & $\mathrm{C} 8$ & $\mathrm{C} 7$ & H5 & -44.3845 \\
\hline 21 & $\mathrm{HCCN}$ & H6 & $\mathrm{C} 8$ & $\mathrm{C} 7$ & $\mathrm{~N}$ & -160.2609 \\
\hline 22 & $\mathrm{CCCC}$ & C9 & $\mathrm{C} 8$ & C7 & C6 & -45.2182 \\
\hline 23 & $\mathrm{CCCH}$ & C9 & $\mathrm{C} 8$ & $\mathrm{C} 7$ & $\mathrm{H} 5$ & -166.0583 \\
\hline 24 & $\mathrm{CCCN}$ & C9 & $\mathrm{C} 8$ & C7 & $\mathrm{N}$ & 78.0654 \\
\hline 25 & $\mathrm{HCCC}$ & $\mathrm{H} 7$ & $\mathrm{C} 8$ & C7 & C6 & -168.9732 \\
\hline 26 & $\mathrm{HCCH}$ & $\mathrm{H} 7$ & $\mathrm{C} 8$ & $\mathrm{C} 7$ & $\mathrm{H} 5$ & 70.1867 \\
\hline 27 & $\mathrm{HCCN}$ & $\mathrm{H7}$ & $\mathrm{C} 8$ & $\mathrm{C} 7$ & $\mathrm{~N}$ & -45.6897 \\
\hline 28 & $\mathrm{HOCC}$ & $\mathrm{H} 4$ & $\mathrm{O} 2$ & C3 & $\mathrm{C} 4$ & 177.5188 \\
\hline 29 & $\mathrm{HOCC}$ & $\mathrm{H} 4$ & $\mathrm{O} 2$ & C3 & $\mathrm{C} 2$ & -2.9488 \\
\hline 30 & $\mathrm{HCCO}$ & $\mathrm{H} 2$ & $\mathrm{C} 4$ & C3 & $\mathrm{O} 2$ & -0.2085 \\
\hline 31 & $\mathrm{HCCC}$ & $\mathrm{H} 2$ & $\mathrm{C} 4$ & C3 & $\mathrm{C} 2$ & -179.7641 \\
\hline 32 & $\mathrm{CCCO}$ & $\mathrm{C} 5$ & $\mathrm{C} 4$ & C3 & $\mathrm{O} 2$ & 179.7198 \\
\hline 33 & $\mathrm{CCCC}$ & $\mathrm{C} 5$ & $\mathrm{C} 4$ & C3 & $\mathrm{C} 2$ & 0.1641 \\
\hline 34 & $\mathrm{HCCC}$ & $\mathrm{H} 2$ & $\mathrm{C} 4$ & $\mathrm{C} 5$ & C10 & 0.9175 \\
\hline 35 & $\mathrm{HCCC}$ & $\mathrm{H} 2$ & $\mathrm{C} 4$ & $\mathrm{C} 5$ & C6 & -179.9801 \\
\hline 36 & CCCC & $\mathrm{C} 3$ & $\mathrm{C} 4$ & $\mathrm{C} 5$ & $\mathrm{C} 10$ & -179.0105 \\
\hline 37 & CCCC & C3 & $\mathrm{C} 4$ & $\mathrm{C} 5$ & C6 & 0.0920 \\
\hline 38 & OCCO & $\mathrm{O} 2$ & $\mathrm{C} 4$ & $\mathrm{C} 2$ & O1 & 0.1050 \\
\hline 39 & $\mathrm{OCCC}$ & $\mathrm{O} 2$ & $\mathrm{C} 4$ & $\mathrm{C} 2$ & $\mathrm{C} 1$ & -179.7159 \\
\hline 40 & $\mathrm{CCCO}$ & $\mathrm{C} 4$ & C3 & $\mathrm{C} 2$ & $\mathrm{O} 1$ & 179.6185 \\
\hline 41 & $\mathrm{CCCC}$ & $\mathrm{C} 4$ & C3 & $\mathrm{C} 2$ & $\mathrm{C} 1$ & -0.2023 \\
\hline 42 & CCCC & C9 & C10 & $\mathrm{C} 5$ & $\mathrm{C} 4$ & 130.6111 \\
\hline 43 & CCCC & C9 & $\mathrm{C} 10$ & C5 & C6 & -48.5230 \\
\hline 44 & HCCC & $\mathrm{H} 10$ & $\mathrm{C} 10$ & $\mathrm{C} 5$ & $\mathrm{C} 4$ & 12.7399 \\
\hline 45 & HCCC & $\mathrm{H} 10$ & $\mathrm{C} 10$ & $\mathrm{C} 5$ & C6 & -166.3942 \\
\hline 46 & CCCC & $\mathrm{C} 12$ & $\mathrm{C} 10$ & $\mathrm{C} 5$ & $\mathrm{C} 4$ & -105.1044 \\
\hline 47 & $\mathrm{CCCC}$ & $\mathrm{C} 12$ & $\mathrm{C} 10$ & $\mathrm{C} 5$ & C6 & 75.7615 \\
\hline 48 & $\mathrm{CCCH}$ & C9 & $\mathrm{C} 10$ & $\mathrm{C} 12$ & H12 & -47.4345 \\
\hline 49 & $\mathrm{CCCH}$ & C9 & $\mathrm{C} 10$ & C12 & H13 & -162.4782 \\
\hline
\end{tabular}



DOI: 10.5281/zenodo.3542089

\begin{tabular}{|c|c|c|c|c|c|c|}
\hline 50 & $\mathrm{CCCC}$ & C9 & $\mathrm{C} 10$ & $\mathrm{C} 12$ & $\mathrm{C} 11$ & 75.1360 \\
\hline 51 & $\mathrm{HCCH}$ & H10 & $\mathrm{C} 10$ & C12 & H12 & 70.5220 \\
\hline 52 & $\mathrm{HCCH}$ & H10 & $\mathrm{C} 10$ & C12 & H13 & -44.5217 \\
\hline 53 & $\mathrm{HCCC}$ & $\mathrm{H} 10$ & $\mathrm{C} 10$ & $\mathrm{C} 12$ & $\mathrm{C} 11$ & -166.9075 \\
\hline 54 & $\mathrm{CCCH}$ & $\mathrm{C5}$ & $\mathrm{C} 10$ & $\mathrm{C} 12$ & H12 & -170.3048 \\
\hline 55 & $\mathrm{CCCH}$ & $\mathrm{C} 5$ & $\mathrm{C} 10$ & C12 & H13 & 74.6515 \\
\hline 56 & CCCC & $\mathrm{C} 5$ & $\mathrm{C} 10$ & $\mathrm{C} 12$ & C11 & -47.7342 \\
\hline 57 & $\mathrm{CCCC}$ & $\mathrm{C} 4$ & $\mathrm{C} 5$ & C6 & $\mathrm{C} 1$ & -0.3172 \\
\hline 58 & CCCC & $\mathrm{C} 4$ & $\mathrm{C} 5$ & C6 & $\mathrm{C} 7$ & 179.6343 \\
\hline 59 & CCCC & $\mathrm{C} 10$ & $\mathrm{C} 5$ & C6 & $\mathrm{C} 1$ & 178.8328 \\
\hline 60 & CCCC & $\mathrm{C} 10$ & $\mathrm{C} 5$ & $\mathrm{C} 6$ & $\mathrm{C} 7$ & -1.2157 \\
\hline 61 & $\mathrm{CCOH}$ & C3 & $\mathrm{C} 2$ & O1 & H3 & 178.1454 \\
\hline 62 & $\mathrm{CCOH}$ & $\mathrm{C} 1$ & $\mathrm{C} 2$ & O1 & H3 & -2.0381 \\
\hline 63 & $\mathrm{CCCC}$ & C3 & $\mathrm{C} 2$ & $\mathrm{C} 1$ & C6 & -0.0181 \\
\hline 64 & $\mathrm{CCCH}$ & C3 & $\mathrm{C} 2$ & $\mathrm{C} 1$ & H1 & 179.8879 \\
\hline 65 & OCCC & $\mathrm{O} 1$ & $\mathrm{C} 2$ & $\mathrm{C} 1$ & C6 & -179.8288 \\
\hline 66 & $\mathrm{OCCH}$ & $\mathrm{O} 1$ & $\mathrm{C} 2$ & $\mathrm{C} 1$ & $\mathrm{H} 1$ & 0.0772 \\
\hline 67 & $\mathrm{CCCC}$ & $\mathrm{C} 5$ & C6 & $\mathrm{C} 1$ & $\mathrm{C} 2$ & 0.2762 \\
\hline 68 & $\mathrm{CCCH}$ & $\mathrm{C} 5$ & C6 & C1 & H1 & -179.6304 \\
\hline 69 & CCCC & $\mathrm{C} 7$ & C6 & $\mathrm{C} 1$ & $\mathrm{C} 2$ & -179.6724 \\
\hline 70 & $\mathrm{CCCH}$ & $\mathrm{C} 7$ & C6 & $\mathrm{C} 1$ & $\mathrm{H} 1$ & 0.4210 \\
\hline 71 & $\mathrm{CCCC}$ & $\mathrm{C} 5$ & C6 & $\mathrm{C} 7$ & $\mathrm{C} 8$ & 49.1511 \\
\hline 72 & $\mathrm{CCCH}$ & $\mathrm{C} 5$ & C6 & $\mathrm{C} 7$ & $\mathrm{H} 5$ & 169.0354 \\
\hline 73 & $\mathrm{CCCN}$ & $\mathrm{C} 5$ & C6 & $\mathrm{C} 7$ & $\mathrm{~N}$ & -73.5733 \\
\hline 74 & $\mathrm{CCCC}$ & $\mathrm{C} 1$ & C6 & $\mathrm{C7}$ & $\mathrm{C} 8$ & -130.8984 \\
\hline 75 & $\mathrm{CCCH}$ & $\mathrm{C} 1$ & C6 & C7 & $\mathrm{H} 5$ & -11.0141 \\
\hline 76 & $\mathrm{CCCN}$ & $\mathrm{C} 1$ & C6 & $\mathrm{C} 7$ & $\mathrm{~N}$ & 10.3772 \\
\hline 77 & $\mathrm{CCNC}$ & $\mathrm{C} 8$ & $\mathrm{C} 7$ & $\mathrm{~N}$ & C11 & -76.2674 \\
\hline 78 & $\mathrm{CCNH}$ & $\mathrm{C} 8$ & $\mathrm{C} 7$ & $\mathrm{~N}$ & H11 & 144.8117 \\
\hline 79 & CCNC & C6 & $\mathrm{C} 7$ & $\mathrm{~N}$ & $\mathrm{C} 11$ & 46.2942 \\
\hline 80 & $\mathrm{CCNH}$ & C6 & $\mathrm{C} 7$ & $\mathrm{~N}$ & H11 & -92.6267 \\
\hline 81 & $\mathrm{HCNH}$ & $\mathrm{H} 5$ & $\mathrm{C} 7$ & $\mathrm{~N}$ & $\mathrm{C} 11$ & 166.2174 \\
\hline 82 & $\mathrm{HCNH}$ & $\mathrm{H} 5$ & $\mathrm{C} 7$ & $\mathrm{~N}$ & H11 & 27.2965 \\
\hline 83 & $\mathrm{CCCN}$ & $\mathrm{C} 10$ & $\mathrm{C} 12$ & $\mathrm{C} 11$ & $\mathrm{~N}$ & -26.4625 \\
\hline 84 & $\mathrm{CCCO}$ & $\mathrm{C} 10$ & $\mathrm{C} 12$ & C11 & $\mathrm{O} 3$ & 158.2655 \\
\hline 85 & $\mathrm{HCCN}$ & H12 & $\mathrm{C} 12$ & $\mathrm{C} 11$ & $\mathrm{~N}$ & 97.0395 \\
\hline 86 & $\mathrm{HCCO}$ & H12 & $\mathrm{C} 12$ & $\mathrm{C} 11$ & $\mathrm{O} 3$ & -78.2325 \\
\hline 87 & $\mathrm{HCCN}$ & H13 & $\mathrm{C} 12$ & C11 & $\mathrm{N}$ & -149.3947 \\
\hline 88 & $\mathrm{HCCO}$ & H13 & $\mathrm{C} 12$ & $\mathrm{C} 11$ & $\mathrm{O} 3$ & 35.3333 \\
\hline 89 & $\mathrm{CNCC}$ & $\mathrm{C} 7$ & $\mathrm{~N}$ & C11 & $\mathrm{C} 12$ & 27.3855 \\
\hline 90 & $\mathrm{CNCO}$ & $\mathrm{C} 7$ & $\mathrm{~N}$ & $\mathrm{C} 11$ & $\mathrm{O} 3$ & -157.0390 \\
\hline 91 & HNCC & H11 & $\mathrm{N}$ & $\mathrm{C} 11$ & $\mathrm{C} 12$ & 165.551 \\
\hline 95 & $\mathrm{HNCO}$ & H11 & $\mathrm{N}$ & $\mathrm{C} 11$ & $\mathrm{O} 3$ & -18.8694 \\
\hline
\end{tabular}


For load analysis of the molecules, the Mulliken population analysis [48], which has its algorithms based on the theory of molecular orbitals, using a linear combination of atomic orbitals (with coefficients determined by the Hartree-Fock method) [49], was used to define a set of molecular orbitals, it is possible to obtain a partition scheme, which distributes the electrons in the atoms, being used to predict and characterize the possible intra and intermolecular interactions of the molecule, being an important descriptor for the correlational study of the structure and the molecular structure. its biological activity [50] [51]. By analyzing the population distribution of charges, Alternamide A showed a variation between atomic charges of atoms of the same element, as the carbon with the highest charge was $\mathrm{C} 14$ with a value of 0.2781 while the lowest charge was the $\mathrm{C} 15$ with a value of 0.2781 from -0.2640 , ranging from 0.5421 load; the same way oxygen with the highest load was $\mathrm{O} 8$ with -0.2349 and the lowest oxygen was 016 with -0.3892 , varying 0.1543; As for hydrogen, atomic charges ranged from 0.1249 in $\mathrm{H} 25$ to 0.2384 in $\mathrm{H} 20$ hydrogen, showing a variation of 0.1135 , inferring that these variations are directly related to the electronegativity differences of atoms (table 7).

Table 7: Mulliken population analysis, showing Mulliken atomic charges for alternamide A atoms $\mathrm{C}, \mathrm{O}$ and $\mathrm{H}$.

\begin{tabular}{|l|l|l|l|}
\hline Atom & Charge & Atom & Charge \\
\hline $01 \mathrm{C}$ & -0.2140 & $16 \mathrm{O}$ & -0.3892 \\
\hline $02 \mathrm{C}$ & -0.0037 & $17 \mathrm{H}$ & 0.1984 \\
\hline $03 \mathrm{C}$ & 0.0706 & $18 \mathrm{H}$ & 0.2209 \\
\hline $04 \mathrm{C}$ & -0.1986 & $19 \mathrm{H}$ & 0.2357 \\
\hline $05 \mathrm{C}$ & -0.0706 & $20 \mathrm{H}$ & 0.2384 \\
\hline $06 \mathrm{C}$ & -0.1294 & $21 \mathrm{H}$ & 0.1370 \\
\hline $07 \mathrm{O}$ & -0.2548 & $22 \mathrm{H}$ & 0.1253 \\
\hline $08 \mathrm{O}$ & -0.2349 & $23 \mathrm{H}$ & 0.1379 \\
\hline $09 \mathrm{C}$ & -0.0514 & $24 \mathrm{H}$ & 0.1253 \\
\hline $10 \mathrm{C}$ & -0.2391 & $25 \mathrm{H}$ & 0.1249 \\
\hline $11 \mathrm{C}$ & -0.2314 & $26 \mathrm{H}$ & 0.1450 \\
\hline $12 \mathrm{C}$ & -0.0883 & $27 \mathrm{H}$ & 0.1400 \\
\hline $13 \mathrm{~N}$ & -0.1207 & $28 \mathrm{H}$ & 0.1522 \\
\hline $14 \mathrm{C}$ & 0.2781 & $29 \mathrm{H}$ & 0.1574 \\
\hline $15 \mathrm{C}$ & -0.2640 & \multicolumn{3}{|l}{} \\
\cline { 1 - 2 } & & &
\end{tabular}

Using the data generated in structural optimization, molecular modeling techniques also contribute to the understanding of intermolecular interactions through the study of electrostatic potential surface maps (MESP) [26] [52]. three-dimensional charge distribution, being important for the analysis and identification of regions with higher and lower electron density, being a strong indicator of nucleophilic and electrophilic sites, being important to analyze descriptors of molecular interactions with polarity and electronegativity, that is, a descriptor capable of assisting in the behavior of molecules against other charged molecules [25][53]. The region with the highest electrostatic potential is indicative of a low negative charge density, which can be understood as a low electron density. Usually, but not always, the color red indicates the lowest potential electrostatic energy, which characterizes a region with higher electron density. The white region represents the largest electrostatic energy, indicating a relative absence of electrons. Intermediate 
colors, green and yellow, represent an intermediate electrostatic potential [54]. Using the threedimensional coordinates of the optimized structure and the charges was rendered the Alternamide A electrostatic potential map, showed a well-wasted distribution of electrostatic potential, where it was possible to identify a higher electron density (nucleophilic region) represented in red, located in the vicinity of oxygen atoms (figure 3), which is indicative of a region in quantity of electrons, being astas the possible places of interaction through electrostatic interactions due to hydrogen bonds.

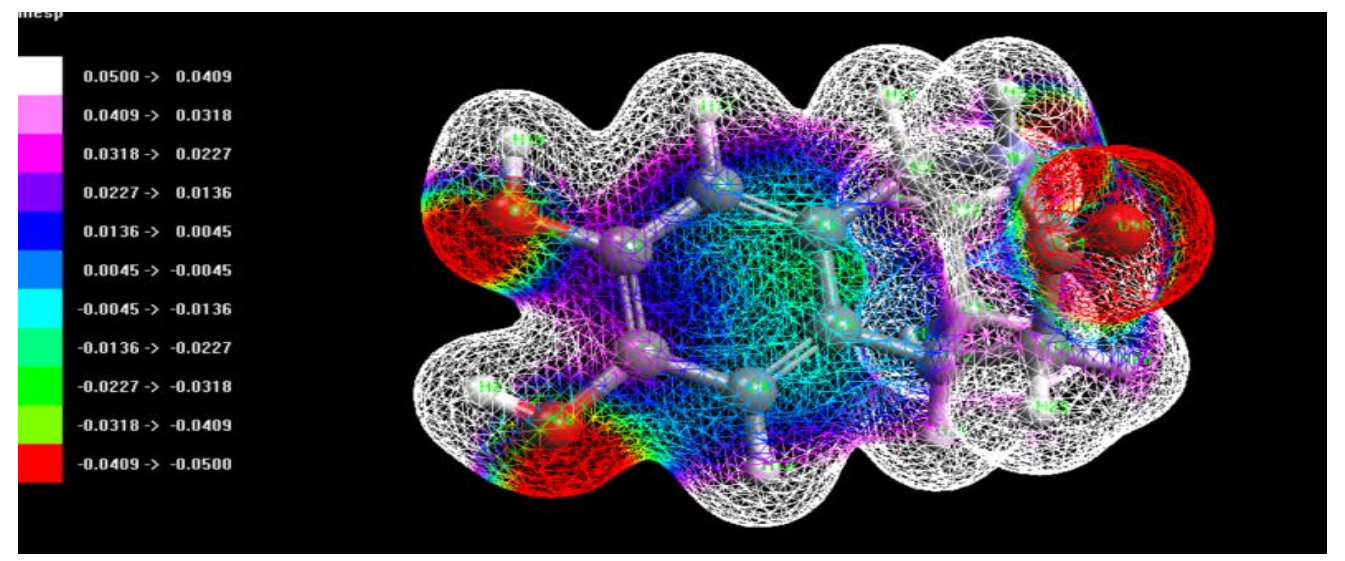

Figure 3: Electrostatic Potential Map rendered from the semi-empirical quantum method using the PM3 parameterization.

Boundary orbitals have been widely related as descriptors of molecule reactivity, because in their theory the transition state formation is due to the interaction between the HOMO (highest occupied molecular orbital) and LUMO (lowest unoccupied molecular orbital) of the species. These are the descriptors that broadly characterize the nature of chemical reactions, since the energy of HOMO is directly related to the susceptibility of the molecule to undergo an electrophilic attack (ionization potential), and the energy of LUMO to susceptibility nucleophile attacks (electron affinity) [55] [56] [57]. In the alternamide molecule it was possible to observe the presence of 77 molecular orbitals and it is possible to identify Homo as number 42, which was symmetrical between its negative (red) and positive (blue) phases, with a strong contribution of oxygen atoms and ligadosbonded atoms el, having an energy of $-0.333062 \mathrm{ev}$. The homo orbital, also symmetrical between the phases, was identified as the orbital 43 , with energy in the order of $-0.002131 \mathrm{eV}$, which is formed by the major contribution of the ligados-bonded atoms present in the ring (figure 4).

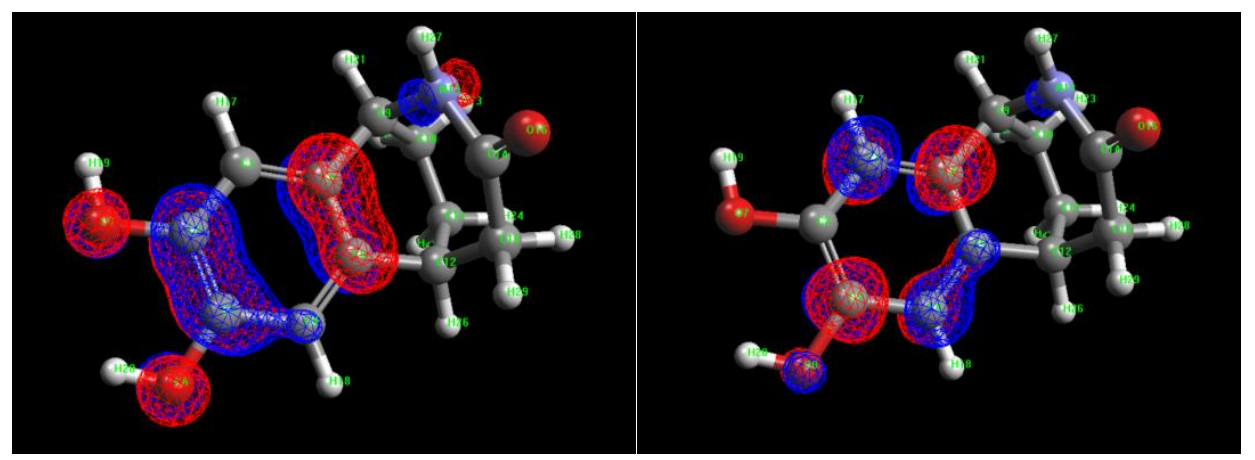

Figure 4: Boundary molecular orbitals, rendered using the semi-empirical quantum method, with parameterization PM3. Fig. 4A (HOMO orbital) and Fig. 4B (LUMO orbital) respectively. 
The GAP energy difference $(\Delta \mathrm{E})$ between HOMO and LUMO orbitals may be related to chemical stability, as a large difference would be indicative of high stability (low reactivity), while a low gap value indicates a high reactivity, as it indicates a low energy value for chemical transition, that is, it indicates the electron-donor and / or electron-acceptor character of a compound [58]. Alternamide had a gap in the order of 0.330931, with ionization potential in the order of To characterize better it was possible to calculate an ionization potential (I) in the order of 0.333062 $\mathrm{eV}$, ie the minimum energy required to remove an electron of alternamide A with respect to energy change when an electron is added to a neutral atom in the gas phase. Electron affinity (A) is described as the change in energy when an electron is added to a neutral atom in the gas phase was predicted to be a small value on the order of $0.002131 \mathrm{eV}$. Noting that low I values may be indicative of a charge transfer mechanism between a ligand-receptor interaction. Another important descriptor to characterize this interaction and electronegativity $(\chi)$, which for alternamide was of the order of $0.167597 \mathrm{eV}$, which is the estimated ability of a molecule to attract electrons from another molecule in a chemical interaction [59].

Using the values of HOMO and Lumo it was possible to calculate the descriptors of polarizability chemical softness (S) 3.021777954, chemical hardness ( $\eta$ ) $0.1665466 \mathrm{ev}$, thus characterizing althernamide $\mathrm{A}$ as a high reactivity molecule, requiring a small amount of energy for a transition of an electron from HOMO to LUMO [60].

Regarding the bioavailability (Figure 5) of the molecule shown in the color zone is the appropriate physicochemical space for oral bioavailability, where the following properties were taken into account as flexibility, lipophilicity, saturation, size, polarity and solubility. The lipophilicity of the studied compound $\log \mathrm{P}$ may range from -0.7 to +5.0 . Molecular weight may range from $150 \mathrm{~g} /$ $\mathrm{mol}$ to $500 \mathrm{~g} / \mathrm{mol}$. The topological polar surface area ranges from 20-130 A². Insolubility was studied using $\log \mathrm{S}$ (ESOL) and ranged from 0 to 6 . The number of rotational bonds should be between $0-9$ and the unsaturation fraction ranges from 0.25 to 1.0 , indicating the fraction of carbon atoms. sp3 hybridization should not be less than 0.25 [61] [62].

The physicochemical properties show that the molecule $\mathrm{C} 12 \mathrm{H} 13 \mathrm{NO}$. The molecular weight was $219.24 \mathrm{~g} / \mathrm{mol}$. The number of heavy atoms is 16 and 6 the number of aromatics. The fraction of carbon atoms in sp3 hybridization was 0.42 . Meanwhile, the number of hydrogen bond acceptors was 3 and the number of hydrogen bond donor was 3. The molar refractivity was 62.34 and the topological polar surface area found was $69.56 \mathrm{~A}^{\circ} 2$.

The $\log \mathrm{Po} / \mathrm{w}(\mathrm{i} \log \mathrm{P})$ is 1.32 , the $\log \mathrm{Po} / \mathrm{w}(\mathrm{X} \log \mathrm{P} 3)$ is 0.61 , the $\log \mathrm{Po} / \mathrm{w}(\mathrm{W} \log \mathrm{P})$ is 0.83 , the $\log \mathrm{Po} / \mathrm{w}(\mathrm{M} \log \mathrm{P})$ is 0.86 the $\log \mathrm{Po} / \mathrm{w}(\mathrm{SILICOS}-\mathrm{IT})$ is 1.32 and the consensus $\log \mathrm{Po} / \mathrm{w}$ is 1.00 respectively. From the $\log \mathrm{P}$ values overall it can be concluded that the compound is having good lipophilic character. The water solubility of the compound was studied using log S (ESOL) value as -1.83 depicting the compound belongs to very soluble water class [63].

The pharmacokinetic properties were studied using the BOILED-Egg model [64] to simulate an intuitive assessment of passive gastrointestinal absorption (HIA) and brain barrier permeation (BBB) as a function of the position of molecules in the WLOGP-versus-TPSA framework (Figure 6). If the substance is in the white region, it indicates that it has a high probability of passive 
absorption by the gastrointestinal tract, whereas if it is in the yellow region, it indicates that it has a high probability of brain permeation [65] [40].

The Alternamide A molecule showed high gastrointestinal absorption, however it does not easily permeate the brain barrier. It is a P-gp substrate meaning there may be problem excreting the drug. For, P-glycoprotein plays a significant role in drug absorption and distortion. Due to its location, P-glycoprotein appears to have a greater impact on limiting drug uptake of blood circulation in the brain and intestinal lumen in epithelial cells than in increasing drug excretion of hepatocytes and renal tubules in the adjacent luminal space. The substance is not an inhibitor of CYP2C19 and CYP2D6 isoenzyme, which means that there is no chance of drug accumulation or interaction resulting in toxicity. The similarity parameter of the drug is high because it follows the rule of Lipinski, Verber, Egan, with a bioavailability score of 0.55. The SWISS ADME Synthetic Accessibility (SA) score is based primarily on the supposed frequency of molecular fragments in obtained molecules correlates with ease of synthesis. The fragmentary contribution to (SA) should be favorable for frequent and unfavorable chemical portions for rare portions. The synthetic accessibility score was 2.75 , which means that the molecule has great viability to be synthesized.

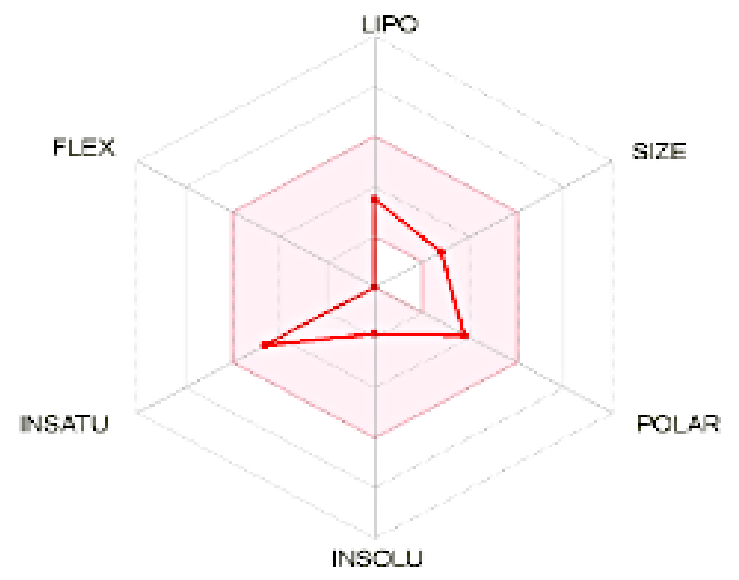

Figure 5: The bioavailability of Alternamide A using Swiss ADME predictor

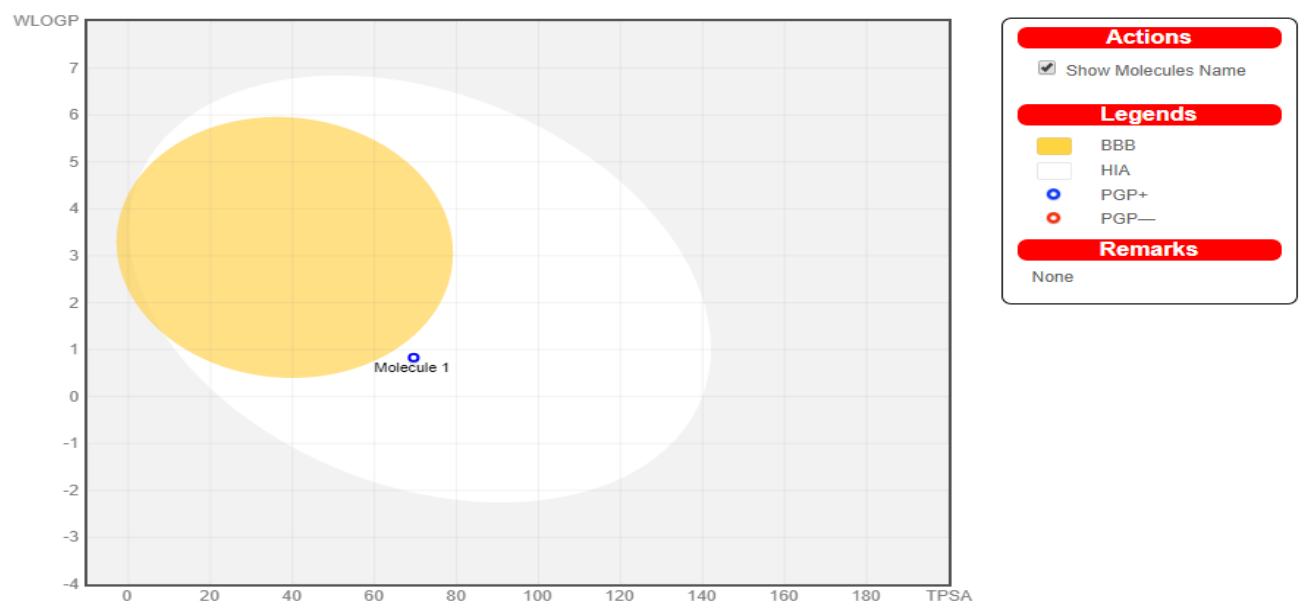

Figure 6: Molecule falling in BOILED-Egg is predicting the molecule is able to penetrate through the blood brain barrier. 


\section{Conclusions}

Using the semi-empirical quantum formalism it was possible to identify the most stable conformation of the tricyclic alkaloid alternamide A, the boundary orbitals, calculate to identify the nucleophilic sites and the reactivity descriptors. Through in silico absorption, distribution, metabolism, excretion and toxicity (ADMET) simulations, including solubility, blood-brain barrier (BBB), plasma protein binding, CYP2D6 binding, gastrointestinal absorption and hepatotoxicity, it was observed that good oral bioavailability and high water solubility high gastrointestinal absorption. The synthetic accessibility score was 2.75 , which means that it would be easy to synthesize the molecule under study. Highlighting what the present study represents as a fundamental step for future molecular docking and drug design studies for the development of T-cruzi evolutionary form inhibitors.

\section{Acknowledgements}

The present work was partially funded by $\mathrm{CNPq}$ - National Council for Scientific and Technological Development and CAPES - Brazilian Federal Agency for Support and Evaluation of Postgraduate Education of the Brazilian Ministry of Education.

\section{References}

[1] Dias, L.C., Dessoy, M.A., Silva JJN, Thiemann OH, Oliva G, Andricopulo AD. Chemotherapy of Chagas' disease: State of the art and perspectives for the development of new drugs. Quim Nova. 2009;

[2] Bermudez, J., Davies, C., Simonazzi, A., Pablo Real, J., Palma, S., Current drug therapy and pharmaceutical challenges for Chagas disease. Acta Tropica. 2016.

[3] Coura, J.R. Ripanosomose, doença de. E N D E M I a S /a R T I G O S. 1999;

[4] De Oliveira Filho, G..B, Cardoso, M.V., Espíndola, J.W..P, Oliveira e Silva, D.A, Ferreira, R.S., Coelho, P.L., et al. Structural design, synthesis and pharmacological evaluation of thiazoles against Trypanosoma cruzi. Eur J Med Chem. 2017;

[5] Repetto, E.C., Zachariah, R., Kumar, A., Angheben, A., Gobbi, F., Anselmi, M., et al. Neglect of a Neglected Disease in Italy: The Challenge of Access-to-Care for Chagas Disease in Bergamo Area. PLoS Negl Trop Dis. 2015;

[6] Moncayo,A., Silveira, A.C. Current epidemiological trends of Chagas disease in Latin America and future challenges: Epidemiology, surveillance, and health policies. In: American Trypanosomiasis Chagas Disease: One Hundred Years of Research: Second Edition. 2017.

[7] De Menezes, R.P.B., Sampaio, T.L., Lima, D.B., Sousa, P.L., De Azevedo, I.E.P., Magalhães, E.P., et al. Antiparasitic effect of $(-)$ - $\alpha$-bisabolol against Trypanosoma cruzi Y strain forms. Diagn Microbiol Infect Dis [Internet]. 2019:114860. Available from: https://doi.org/10.1016/j.diagmicrobio.2019.06.012

[8] Koolen, H.H.F., Pral, E.M.F., Alfieri, S.C., Marinho, J.V.N., Serain, A.F., Hernández-Tasco, A.J.,. Antiprotozoal and antioxidant alkaloids from Alternanthera littoralis. Phytochemistry. 2017;

[9] Lima, A.R., Silva, J., Bezerra, L.L., Marinho, M.M., Marinho, E.S. Molecular docking of potential curcuminoids inhibitors of the NS1 protein of dengue virus. Int J Sci Eng Res. 2017;8(4).

[10] Carneiro, S.S., Lima, A.R., Marinho, M.M., Marinho, E.S. In silico Study Of The Therapeutic Agent In The Treatment Of Non-Hodgkin's Lymphomas, Peripheral T- Cell Belinostat, A SemiEmpirical Approach. Imp J Interdiscip Res. 2016;(8):1645-8. 
[11] Oliveira, V.M. De, Marinho, M.M., Marinho, E.S., Semi-Empirical Quantum Characterization of the Drug Selexipag: HOMO and LUMO and Reactivity Descriptors. Int J Recent Res Rev. 2019; XII (2):15-20.

[12] Henrique, C., Roberto, A., Marinho, E.S., Campos, O.S., Nithael,. F, Lucio M, Characterization of the natural insecticide methylcytisine: An in silico study using classic force field. Int J Recent Res Rev. 2019; XII (2):15-20.

[13] Cheng, F., Li, W., Zhou, Y., Shen, J., Wu, Z., Liu, G., et al. AdmetSAR: A comprehensive source and free tool for assessment of chemical ADMET properties. J Chem Inf Model. 2012;

[14] Tareq-Hassan Khan, M. Predictions of the ADMET Properties of Candidate Drug Molecules Utilizing Different QSAR/QSPR Modelling Approaches. Curr Drug Metab. 2010;

[15] Chinthala, Y., Thakur, S., Tirunagari, S., Chinde, S., Domatti, A.K., Arigari, N.K., et al. Synthesis, docking and ADMET studies of novel chalcone triazoles for anti-cancer and anti-diabetic activity. Eur J Med Chem. 2015;

[16] Atkins,. P, De Paula, J. Atkins' physical chemistry / Peter Atkins, Julio de Paula. Physical chemistry. 2010.

[17] Lopes, D.,Oliveira, S. De, Marinho, M.M., Marinho, E. S., Butanamide. Characterization in Silic of Anti-Epiletic Drug ( 2S ) -2- [( 4R ) -2- O--propylpyrrolidin--yl,. 2018; XI(4):5-12.

[18] Csizmadia, P. MarvinSketch and MarvinView: Molecule Applets for the World Wide Web. In 2019.

[19] Thompson, M.A., ArgusLab 401. Planaria Software LLC, Seattle, WA. ArgusLab 4.0. 1. Seattle; 2010.

[20] Thompson, J.D., Cramer, C.J., Truhlar, D.G. Parameterization of charge model 3 for AM1, PM3, BLYP, and B3LYP. J Comput Chem. 2003;

[21] Hanwell, M.D., Curtis, D.E., Lonie, D.C., Vandermeerschd, T., Zurek, E., Hutchison, G.R. Avogadro: An advanced semantic chemical editor, visualization, and analysis platform. J Cheminform. 2012;

[22] Reges, M., Marinho, M.M., Marinho, E.S. Semi-Empirical Study of the Drug Riociguat , an Important Drug for Oral Treatment against Chronic Thromboembolic Pulmonary Hypertension. Int J Sci Eng Sci. 2017;1(1):13-7.

[23] Araujo, G.A, Silva, E.P., Sanabio, R.G., Pinheiro, J.A., Albuquerque, M.B., Castro, R.R., et al. Characterization in Silico of the Structural Parameters of the Antifungal Agent Ketoconazole. Biol Chem Res. 2016;

[24] Paes, L., Santos, W.L., Marinho, M.M., Marinho, E.S. ESTUDO DFT DO ALCALOIDE DICENTRINA: GAP, HOMO, LUMO, MESP E MULLIKEN. JOIN. 2017;(1).

[25] Prabavathi, N., Nilufer, A., Krishnakumar, V. Molecular structure, vibrational, UV, NMR, hyperpolarizability, NBO and HOMO-LUMO analysis of Pteridine2,4-dione. Spectrochim Acta Part A Mol Biomol Spectrosc. 2012;

[26] Mulliken, R.S. Electronic population analysis on LCAO-MO molecular wave functions. I. J Chem Phys. 1955;

[27] Atkins, P.W, Overton, T., Rourke, J., Weller, M., Armstrong, F., Hagerman, M. Shriver \& Atkins' Inorganic Chemistry. Shriver and Atkin's inorganic chemistry. 2010.

[28] Gopakumar, T.G., Meiss, J., Pouladsaz, D., Hietschold, M. HOMO-LUMO gap shrinking reveals tip-induced polarization of molecules in ultrathin layers: Tip-sample distance-dependent scanning tunneling spectroscopy on d8 (Ni, Pd, and Pt) phthalocyanines. J Phys Chem C. 2008;

[29] Suresh, Kumar. S., Athimoolam, S., Sridhar, B. XRD, vibrational spectra and quantum chemical studies of an anticancer drug: 6-Mercaptopurine. Spectrochim Acta - Part A Mol Biomol Spectrosc. 2015;

[30] Koopmans, T. Über die Zuordnung von Wellenfunktionen und Eigenwerten zu den Einzelnen Elektronen Eines Atoms. Physica. 1934; 
[31] Vijayaraj, R., Subramanian, V., Chattaraj. P.K. Comparison of global reactivity descriptors calculated using various density functionals: A QSAR perspective. J Chem Theory Comput. 2009;

[32] Chermette, H. Chemical reactivity indexes in density functional theory. J Comput Chem. 1999;

[33] Padmanabhan, J., Parthasarathi, R., Subramanian, V., Chattaraj, P.K. Electrophilicity-based charge transfer descriptor. J Phys Chem A. 2007;

[34] Chattaraj, P.K., Sarkar, U. Theoretical Aspects of Chemical Reactivity. Theoretical and Computational Chemistry. 2007.

[35] Parr, R.G., Pearson RG. Absolute hardness: companion parameter to absolute electronegativity. J Am Chem Soc [Internet]. 1983 Dec;105(26):7512-6. Available from: http://pubs.acs.org/doi/abs/10.1021/ja00364a005

[36] Parr, R.G., Chattaraj, P.K. Principle of Maximum Hardness. J Am Chem Soc. 1991;

[37] Lee, C., Yang, W., Parr, R.G. Into a Functional of the Electron Density F F. Phys Rev B. 1988;

[38] Okoli, P.T, Nzute, V.C, Durojaye, O.A., Chielo, O.H., Ajibo, Q.C., Udo, S.I., et al. An In-silico Pharmacokinetics Study on Cis- A Nutraceutical Compound with Anticancer Properties. 2019;7(3):1-7.

[39] Silva, A.B.F, Marinho M.M., Mendes F.R.D.S. In Silico Study of Phytochemical Chlorogenic Acid: A Semi- Empirical Quantum Study and Adme. Int J Recent Res Rev. 2019; XII (2):34-9.

[40] Daina, A., Michielin, O., Zoete, V. SwissADME: A free web tool to evaluate pharmacokinetics, drug-likeness and medicinal chemistry friendliness of small molecules. Sci Rep. 2017;

[41] Wildman, S.A, Crippen, G.M. Prediction of physicochemical parameters by atomic contributions. J Chem Inf Comput Sci. 1999;

[42] Lipinski, C.A, Lombardo, .F, Dominy, B.W., Feeney, P.J. Experimental and computational approaches to estimate solubility and permeability in drug discovery and development settings. Advanced Drug Delivery Reviews. 2012.

[43] Waring, M.J. Defining optimum lipophilicity and molecular weight ranges for drug candidatesMolecular weight dependent lower log D limits based on permeability. Bioorganic Med Chem Lett. 2009;

[44] Lewars, E.G. Computational chemistry: Introduction to the theory and applications of molecular and quantum mechanics: Third Edition 2016. Computational Chemistry: Introduction to the Theory and Applications of Molecular and Quantum Mechanics: Third Edition 2016. 2016.

[45] Marinho, E.S. Utilização Do Método Semi-Empírico Pm7 Para Caracterização Do Fármaco Atalureno : Homo ,. Rev Expressão Católica. 2016;1(1):177-84.

[46] Atkins, P., Paula, J. de, Friedman, R.. Quanta, Matter, and Change: A molecular approach to physical chemistry. New York. 2009;

[47] Atkins, P., Friedman, R., Molecular Quantum Mechanics Fourth Edition. Oxford Univ Press New York. 2005;

[48] Eryilmaz, S., Gül, M., Inkaya, E., Taş, M. Isoxazole derivatives of alpha-pinene isomers: Synthesis, crystal structure, spectroscopic characterization (FT-IR/NMR/GC-MS) and DFT studies. J Mol Struct. 2016;

[49] Morgon, N.H., Custodio, R., Custódio, R., Morgan, N. H. and Custódio, R. Teoria do Funcional da Densidade. Quim Nov [Internet]. 1995;18(1):44. Available from: http://quimicanova.sbq.org.br/qn/qnol/1995/vol18n1/v18_n1_10.pdf

[50] Reges, M., Marinho, M.M., Marinho, E.S. Structural Characterization of the Hypoglycemic Drug Glimepiride. Int J Recent Res Rev. 2018; XI (2):26-35.

[51] Carneiro, S.S., Marinho, M.M., Marinho, E.S. Electronic / Structural Characterization of Antiparkinsonian Drug Istradefylline: A Semi-Empirical Study. Int J Recent Res Rev. 2017; X (4):9-14.

[52] Marinho ES. A DFT study of synthetic drug topiroxostat: MEP, HOMO, LUMO. Int J Sci Eng Res. 2016;7(July):1264-70. 
[53] Eyre, R.J., Goss, J..P, MacLeod, R.M., Briddon, P.R. Stability of singly hydrated silanone on silicon quantum dot surfaces: Density functional simulations. Phys Chem Chem Phys. 2008;

[54] Mohan, N., Suresh, C.H. A molecular electrostatic potential analysis of hydrogen, halogen, and dihydrogen bonds. J Phys Chem A. 2014;

[55] Lai, T.Y, Guo, J.D., Fettinger, J.C., Nagase, S., Power, P.P. Facile insertion of ethylene into a group 14 element-carbon bond: effects of the HOMO-LUMO energy gap on reactivity. Chem Commun. 2019;

[56] Rottschäfer, D., Sharma, M.K., Neumann, B., Stammler, H.G., Andrada, D.M., Ghadwal, R,S. A Modular Access to Divinyldiphosphenes with a Strikingly Small HOMO-LUMO Energy Gap. Chem - A Eur J. 2019;

[57] AlAbbad, S., Sardot, T., Lekashvili, O., Decato, D., Lelj F., Ross J.B.A., et al. Trans influence and substituent effects on the HOMO-LUMO energy gap and Stokes shift in Ru mono-diimine derivatives. J Mol Struct. 2019;

[58] The Theoretical Prediction of Thermophysical properties, HOMO, LUMO, QSAR and Biological Indics of Cannabinoids (CBD) and Tetrahhdrocannabinol (THC) by Computational Chemistry. Adv J Chem A. 2019;

[59] Arroio, A., Honório, K.M., Da Silva, A.B.F. Propriedades químico-quânticas empregadas em estudos das relações estrutura-atividade. Quimica Nova. 2010.

[60] Rajan, V.K., Muraleedharan, K. A computational investigation on the structure, global parameters and antioxidant capacity of a polyphenol, Gallic acid. Food Chem [Internet]. 2017;220:93-9. Available from: http://dx.doi.org/10.1016/j.foodchem.2016.09.178

[61] Ferreira, L.L.G, Andricopulo, A.D. ADMET modeling approaches in drug discovery. Drug Discovery Today. 2019.

[62] Vora, J., Patel, S., Sinha, S., Sharma, S., Srivastava, A., Chhabria, M., et al. Molecular docking, QSAR and ADMET based mining of natural compounds against prime targets of HIV. J Biomol Struct Dyn. 2019;

[63] Zapadka, M., Kaczmarek, M., Kupcewicz, B., Dekowski, P., Walkowiak, A., Kokotkiewicz, A., et al. An application of QSRR approach and multiple linear regression method for lipophilicity assessment of flavonoids. J Pharm Biomed Anal. 2019;

[64] Daina, A., Zoete, V. A BOILED-Egg To Predict Gastrointestinal Absorption and Brain Penetration of Small Molecules. ChemMedChem. 2016;

[65] Singh, G., Satija, P., Singh, B., Sinha, S., Sehgal, R., Sahoo, S.C. Design, crystal structures and sustainable synthesis of family of antipyrine derivatives: Abolish to bacterial and parasitic infection. J Mol Struct. 2020;

\footnotetext{
*Corresponding author.

E-mail address: Emmanuel.marinho@ uece.br
} 